ESDA2012-82460

\title{
REAL-TIME CONTROL OF THE HEATING OF AN AIRFOIL
}

\author{
Francoise Rétat-Masson \\ GEM - Ecole Centrale Nantes \\ BP 92101- 44321 Nantes \\ France
}

\author{
Elias Cueto \\ Aragon Institute of \\ Engineering Research \\ Universidad de Zaragoza \\ 50018 Zaragoza- Spain
}

\author{
Francisco Chinesta \\ EADS Foundation Int. Chair \\ GEM - Ecole Centrale Nantes \\ BP 92101- 44321 Nantes -France
}

\author{
Adrien Leygue \\ EADS Foundation Int. Chair \\ GEM, UMR CNRS (E C Nantes) \\ BP 92101- Nantes -France
}

\author{
Laurent Dala \\ School of Mechanical, Industrial \\ and Aeronautical Engineering \\ University of the Witwatersrand \\ Johannesburg, South Africa
}

\author{
Craig Law \\ School of Mechanical, Industrial \\ and Aeronautical Engineering \\ University of the Witwatersrand \\ Johannesburg, South Africa
}

\section{EXTENDED ABSTRACT}

Dynamic Data-Driven Application Systems constitute nowadays one of the most challenging applications of simulation-based Engineering Science [1]. DDDAS imply a set of techniques that allow the linkage of simulation tools with measurement devices for real-time control of systems and processes [2]. DDDAS entails the ability to dynamically incorporate additional data into an executing application, and in reverse, the ability of an application to dynamically steer the measurement process. These systems need accurate and fast simulation tools, hence the off-line computations to limit as much as possible the on-line computations. In order to obtain the most efficient solver, all the sources of variability are introduced as extra-coordinates as to solve only once the model off-line to obtain its most general solution to be then considered in on-line purpose. However, such models result defined in highly multidimensional spaces. A technique recently proposed, called Proper Generalized Decomposition [3], allows circumventing this redoubtable curse of dimensionality.

The main driver for this research is the development of new boundary control methods. on an airfoil. This would influence the laminar-turbulent transition and boundary layer separation, allowing an improvement in economic efficiency and safety of airplanes. This new approach proposed is associated with the unsteady surface heating regime using electrically resistant strips embedded in the wing skin. The control of the boundary layer separation and transition will provide a reduction in friction drag, and hence a reduction in the fuel consumption. The implementation of strips in the wing skin could be done at a low cost for the manufacturer without weakening the structural integrity of the wing.

The experiments will be conducted in the transonic wind tunnel at the University of the Witwatersrand. Two sets of model will be manufactured : a metallic and a composite wing. One of the critical parameters of the research will be the transition detection on the wing profiles. It is proposed to use infrared thermography and hot films distributed along the chord in order to detect the boundary layer transition.

Active thermal control consists in applying the required thermal power $f(x)$ (the power being different along the strip) in order to ensure the desired temperatures on several points of the aircraft wing. As during a flight the flow conditions or the ambient temperatures vary, the thermal power $f(x)$ changes and must be chosen as fast as possible in order to ensure optimal operating conditions.

In what follows, for simplicityô sake, we assume that the heat transfer equation is defined in a one dimensional domain of length L, and for now, we consider a quasi-static evolution, which gives us the following steady state heat transfer equation:

$$
k \frac{d^{2} u}{d x^{2}}=f(x), \quad x \in \Omega_{x}=(0, L)
$$


where $\mathrm{k}$ denotes the thermal conductivity, and $\mathrm{f}(\mathrm{x})$ represents the heat source term.

The solution of the heat equation requires to define appropriate boundary conditions at $\mathrm{x}=0$ and $\mathrm{x}=\mathrm{L}$. As we are interested in the control of the temperature of certain points on the airfoil, we are considering the following convection boundary conditions :

$$
\left\{\begin{array}{l}
-\left.k \frac{d u}{d x}\right|_{x=0}=h_{l}\left(u_{a m b}^{l}-u_{(x=0)}\right) \\
\left.k \frac{d u}{d x}\right|_{x=L}=h_{r}\left(u_{a m b}^{r}-u_{(x=L)}\right)
\end{array}\right.
$$

where the convection coefficients $h_{1}$ and $h_{r}$ depend on the flow regime on the left and right borders respectively, and $u_{a m b}^{l}$ and $u_{a m b}^{r}$ are the surrounding ambient temperature at the borders.

At this stage of the research, we chose the simplest source term possible. $f(x)=$ a being too restrictive, because for example, in the case of symmetrical configurations, we obtain : $\mathrm{u}_{\mathrm{x}=0}=\mathrm{u}_{\mathrm{x}=\mathrm{L}}$. As we are interested in controlling two different temperatures $\tilde{u}_{l} \neq \tilde{u}_{r}$, the simplest choice for the source term is :

$$
f(x)=\chi_{l}(x) \cdot a+\chi_{r}(x) \cdot b
$$

where $\mathrm{a}$ and $\mathrm{b}$ are the different power rates applied on the two regions of the domain defined by $\chi_{l}(x)$ and $\chi_{r}(x)$ :

$$
\chi_{l}(x)=\left\{\begin{array}{rr}
1 & x<\frac{L}{2} \\
0 & x>\frac{L}{2}
\end{array} \text { and } \chi_{r}(x)=\left\{\begin{array}{rr}
0 & x<\frac{L}{2} \\
1 & x>\frac{L}{2}
\end{array}\right.\right.
$$

We are looking for the values of $(a, b)$ which would give us the wanted temperatures $\tilde{u}_{l}$ and $\tilde{u}_{r}$ at the borders of the domain.

To ensure the real-time response, we firstly solve the problem once and for all off-line. We must then define a cost function that will lead us to find the optimal operating conditions as fast as possible, as these conditions will change the moment after. Only this last calculus will be done on-line.

In order to find the optimal $(a, b)$, we start with trial values $\left(\mathrm{a}^{\text {trial }}, \mathrm{b}^{\text {trial }}\right)$. We then compute a cost function $\mathrm{C}\left(\mathrm{a}^{\text {trial }}, \mathrm{b}^{\text {trial }}\right)$ which measures the difference with respect to the optimal conditions :

$$
\begin{aligned}
\mathrm{C}\left(\mathrm{a}^{\text {trial }}, b^{\text {trial }}\right)= & \left(u\left(x=0 ; a^{\text {trial }}, b^{\text {trial }}\right)-\tilde{u}_{l}\right)^{2} \\
& +\left(u\left(x=L ; a^{\text {trial }}, b^{\text {trial }}\right)-\tilde{u}_{r}\right)^{2}
\end{aligned}
$$

which is null if and only if $u(x=0)=\tilde{u}_{l}$ and $u(x=L)=\tilde{u}_{r}$.
If the cost function for these trial values is not null, $\left(\mathrm{a}^{\text {trial }}, \mathrm{b}^{\text {trial }}\right)$ must be updated, using any appropriate optimization strategy.

In a first step, we solved the problem with only 3 coordinates (for $\mathrm{x}$, $\mathrm{a}$ and $\mathrm{b}$ ) using a traditional discretizing technique (in our case, finite differences), i.e. we solved the temperature field, for any value of $\mathrm{a} \in \Omega_{\mathrm{a}}$ and $\mathrm{b} \in \Omega_{\mathrm{b}}$. We then looked for all $\left(\mathrm{a}_{\mathrm{l}}, \mathrm{b}_{\mathrm{l}}\right)$, which satisfied the left border condition $\mathrm{u}_{\mathrm{x}=0}=\tilde{u}_{l}$. We proceeded with looking for all $\left(a_{r}, b_{r}\right)$, which satisfied the right border condition $\mathrm{u}_{\mathrm{x}=\mathrm{L}}=\tilde{u}_{r}$. The intersection of $\left(\mathrm{a}_{\mathrm{l}}, \mathrm{b}_{\mathrm{l}}\right)$ and $\left(\mathrm{a}_{\mathrm{r}}, \mathrm{b}_{\mathrm{r}}\right)$ gave us the final solution $\left(a_{1 r}, b_{l r}\right)$. A faster way of obtaining the same final solution $\left(\mathrm{a}_{\mathrm{lr}}, \mathrm{b}_{\mathrm{lr}}\right)$ consists in using Newton $\hat{Q}$ method to solve the system of equation deriving from the cost equation :

$$
\left\{\begin{array}{l}
R_{l}=u\left(x=0, a_{l r}, b_{l r}\right)-\tilde{u}_{l} \\
R_{r}=u\left(x=L, a_{l r}, b_{l r}\right)-\tilde{u}_{r}
\end{array}\right.
$$

But as the problemô dimension becomes higher (introducing all the external parameters as extra-coordinates thermal conductivity, ambient temperature, convection coefficients é ), it is no longer possible to use a traditional technique to solve the thermal problem for each possible combination of all the parameters. So we introduce the PGD to circumvent the curse of dimensionality [4]. The PGD method consists of finding a solution assuming a separated representation, which in our case would be :

$$
u(x, a, b) \approx \sum_{k=1}^{k=N} X_{i}(x) \cdot A_{i}(a) \cdot B_{i}(b)
$$

Thanks to this separated representation, the complexity scales linearly with the number of parameters considered as extra-coordinates, instead of the exponential growth that characterizes traditional discretizing techniques.

\section{REFERENCES}

1. J.T. Oden, T. Belytschko, J. Fish, T.J.R. Hughes, C. Johnson, D. Keyes, A. Laub, L. Petzold, D. Srolovitz and S. Yip. Simulation-based Engineering Science : Revolutionizing Engineering Science trough simulation. NSF Blue Ribbon Panel on SBES. 2006

2. NSF Final Report. DDDAS Workshop 2006, Arlington, VA, USA.2006.

3. F.Chinesta, A. Ammar, E. Cueto. Recent advances in the use of the Proper Generalized Decomposition for solving multidimensional models. Archive of Computational Methods in Engineering ï State of the Art Reviews, 2010.

4. F. Chinesta, A. Ammar, E. Cueto, Recent advances in the use of the Proper Generalized Decomposition for solving multidimensional models, Archives of Computational Methods in Engineering, 17, 4, 327-350, 2010. 